\title{
Research on a Sensor Network Communication Optimization Algorithm
}

\author{
Shi Yanjie, Yu Lina \\ Hebei College of Industry and Technology \\ Shijiazhuang Hebei 050000 China
}

\begin{abstract}
The peripheral malfunction will cause the nodes fail to work when there is no central control node in the large scale sensor network. The network can't guarantee the continuity of the communication and service and the communication efficiency will be low in this situation. In order to fix the issue, the paper proposes a correcting fusion algorithm for the sensor network data communication. When the collision of the communication data happens in the sensor network due to the failure of the nodes, the correcting fusion algorithm can ensure the effective data fusion between the fault nodes and other nodes to guarantee the communication to the maximum extent. The experiment indicates the algorithm can increase the efficiency of the sensor network to achieve the satisfactory results.
\end{abstract}

Keywords- Communication Optimization; Sensor Network; Fusion algorithm

\section{INTRODUCTION}

The Wireless Sensor Network is consist of large amount of energy constrain nodes[1]. The data fusion problem is one the main research issue in the Wireless Sensor Network research[2]. Current wireless sensor network data fusion algorithm is based on the $\mathrm{C} / \mathrm{S}$ model. There are many disadvantages of the model. First, the delay and energy consumption in the network will increase with the number of nodes increasing; second, the scalability is not good and the network structure will be adjusted when there is new node; third, the energy among the nodes is extremely imbalance[3]. Recently, the Mobile Agent (MA) computation model fixes the advantages in the $\mathrm{C} / \mathrm{S}$ model to some extent. Some researchers following proposed MA data fusion algorithm based on local shortest priority. When the network area is large and the distribution of the nodes is complex, the algorithm can only find the shortest local shortest routing path, thus it's merely suitable for small scale sensor network in simple situation[4]. With the mature of heuristic algorithm, many researchers proposed MA data fusion techniques based on genetic algorithm. However, the generic algorithm fusion energy is the function of time which can't reduce the data transmission, so the energy can't be saved and it has lots of shortcomings[5]. This paper proposes a correcting fusion algorithm for sensor network data communication. When the collision of the communication data happens in the sensor network due to the failure of the nodes, the correcting fusion algorithm can ensure the effective data fusion between the fault nodes and other nodes to guarantee the communication to the maximum extent.

\section{FUSION ALGORITHM BASED ON THE MASSIVE DATA COMMUNICATION ERROR CORRECTION}

For the traditional sensor network data communication method, the communication capacity of each node should be detected before communication. The data capacity $\mathrm{M}$ of each node is different in the network communication. Thus the communication data capacity of each node is distinct. The available data capacity $M$ of each node should be computed which can be described in capacity weigh. The $M$ can be computed by traversing each different node in the network. The method is as follow:

\section{A. Compute the node capacity information $M$}

Marking each node and edge and assuming there is $n$ nodes and $\mathrm{m}$ edges in the network, the weigh of each edge can be computed. The formula is:

$$
M_{i}=\frac{Q D_{i} \log \left(\frac{1}{D_{i}}+0.01\right)}{\sqrt{\sum_{j=1}^{M} D_{i j}{ }^{2} \log ^{2}\left(\frac{1}{D_{i j}}+0.01\right)}}
$$

In the above equation, $M_{i}$ is the information capacity of the nodes in $\mathrm{i}$ th path; $\mathrm{Q}$ is the number of data communication nodes in the equivalent network; $D_{i}$ is the node to node distance for communication data; $D_{i j}$ is the node to node communication distance and also data communication distance. Assuming there are multiple nodes in the equivalent networks, they can form a multi-path network. Each node can compute the data capacity.

\section{B. Massive data communication correction method}

After computing the capacity coefficient of the network nodes, the computed capacity can be used to communicate. The ability to communicate depends on the capacity computed. However, with the increase of data, some errors can't be avoided to happen in the data communication. Once the error happens, the data will collide and affect the next node's communication. It's assumed that one node in the network has over flow error and the communication between nodes is one way. If the communication relationship between the communication node $v_{i}$ and the nearby direct $v_{j}$ should be established, the communication exclusive error weigh can be calculated which is indicated by $\mathrm{W}\left(v_{i}, v_{j}\right)$; if there 
is no relationship between the data communication node $v_{i}$ and other node $v_{j}$, the data communication error correlation is not 0 which is quite small compare to nearby nodes. The data capacity between two nodes is the basic of computing the correcting ability.

(1) Apply following formula to calculate data communication error probability of each node. The method is following:

$$
I\left(v_{i}, e_{j} \mid c\right)=\sum_{v_{i}, v_{j}, c} p\left(v_{i} v_{j} \mid c\right) \log \frac{p\left(v_{i}, v_{j} \mid c\right)}{p\left(v_{i} \mid c\right) p\left(v_{j} \mid c\right)}
$$

In the formula, $v_{i}$ is the capacity information of node $\mathrm{i}, \mathrm{c}$ is the empirical coefficient indicating the degree of smooth.

(2) Based on the information of all of the nodes in the equivalent network, the network node communication diagram can be built. The communication error computing weigh $I\left(v_{i}, e_{j} \mid c\right)$ can be calculated for each pair of equivalent nodes $\left(x_{i}, x_{j}\right)$.

(3) When arbitrarily selects a node as the root, all none directs can be converted to the direct edge point to the neighbor to form the tree BT.

(4) The class node C is set as the farther with all characteristics to join in the tree BT to form graph G.

(5) The correcting can be formed by computing each node's communication error probability to change the communication threshold to ensure the error probability is least.

Applying the above computational method to calculate the communication data error probability, the optimal points between efficiency and accuracy is set. The advantage of tree enhanced communication classifier is that the time complex rate of learning algorithm is less than typical classifier. The disadvantage is that it fails to consider the correlation among the characteristics which constrains its application.

\section{Distinct data fusion communication}

If there are differences among pending communication data, the collision easily happens which will lower the communication efficiency. The efficiency of the communication data will be improved by using data fusion technique to classify the data into different types. The available information in the pending communication data will distribute in a specific area which depends on the data frequency. The difference among the data can be distinguished by variance, which is caused by distinct types of signal. The fusion ability of the fused data is indicated as follows.

$$
C_{j, k}^{S}(p)=\frac{d_{j, k}^{S}(p)}{\sum_{q \in r_{i}^{F}} a_{j}^{S}(q)}
$$

In the equation, $p$ is $p \in r_{i}^{F}$ in which $j, k, p$ is related probability, $i=1,2, \cdots, k_{F}, q \in r_{i}^{F}$ indicates $\mathrm{q}$ is belong to the information class $r_{i}^{F}$, the information fusion coefficient can be calculated as follows.

$$
\begin{aligned}
& d_{j, k}^{F}(p)= \begin{cases}d_{j, k}^{A}(p), & \text { if } C_{j, k}^{A}(p) \succ C_{j, k}^{B}, p \in r_{i}^{F} \\
d_{j, k}^{B}(p), & \text { if } C_{j, k}^{A}(p) \leq C_{j, k}^{B}, p \in r_{i}^{F}\end{cases} \\
& d_{j, k}^{F}(p) \text { is the network information fusion coefficient. }
\end{aligned}
$$

The fusion coefficient can be set depending on different noise data in distinct situation. The noise fusion coefficient is following when the noise is small.

$$
S_{j}^{c}(F, k)=\max _{q \in Q}\left(\left|D_{j}^{\varepsilon}(F, q)\right|\right)
$$

In the above equation, $\mathrm{j}$ is serial number of the network. $\varepsilon=1,2,3$ communication, $k=(m, n)$ is a kind of network data.

The above method can be applied to fix the data over flow in the sensor network and the data error in the collision to ensure the efficiency of data communication and effective communication.

\section{EXPERIMENT RESULTS AND ANALYSIS}

In order to justify the algorithm in this paper, Matlab 7.1 is applied to simulate the experiment. The wireless sensor network is deployed in the $100 \mathrm{~m} \times 100 \mathrm{~m}$ square and the nodes is distributed randomly. The amount is between 50 and 300 . The communication radius of the node is $50 \mathrm{~m}$, the sink node is fixed in the left below as shown in figure 1 ..

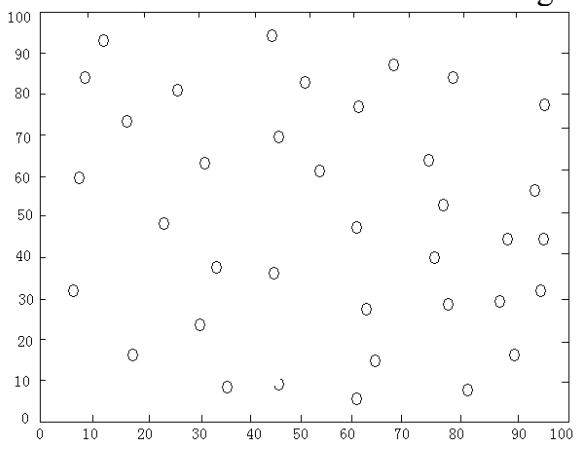

Figure 1. The deployment of the nodes in the wireless sensor network

\section{A. Network energy consumption analysis}

In the Mobile Agent wireless sensor network the network energy consumption is consist of two parts. Fist, it's the consumption for mobile planning path to evaluate the path cost which indicates the resource cost of the path; second, it the information gain energy consumption in optimizing the path. In typical situation the unit information gain cost is evaluated by the ratio between the costs in mobile planning and the costs in the whole information gain. Figure 5 shows the costs of unit information gain in different scale of nodes for the two algorithms. 


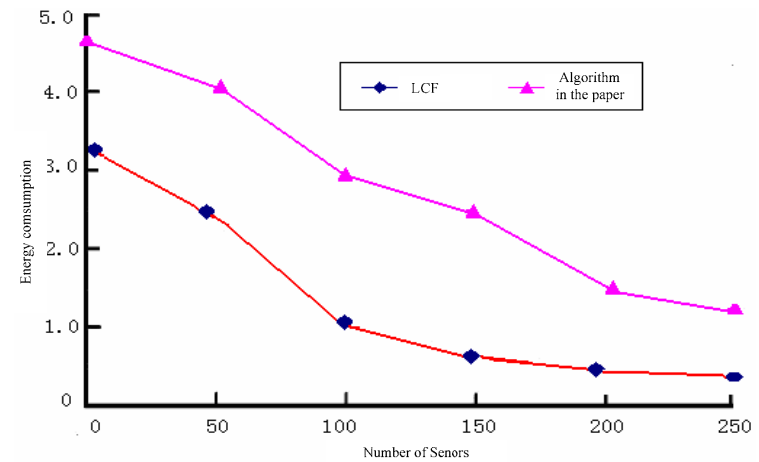

Figure 2. The comparison of the network energy consumption

In figure 5, the traditional algorithm is worse than the particles algorithm .Thus in the same situation the algorithm proposed in the paper can prolong the lifetime of the network.

\section{B. Network delay analysis}

The network delay begins when the sink node starts to dispatch mobile agents in the wireless sensor network based on the mobile agents. It is the time for the sink node when the mobile agents return the fusion data. Figure 6 shows the network delay of the two algorithms with the increase of nodes.

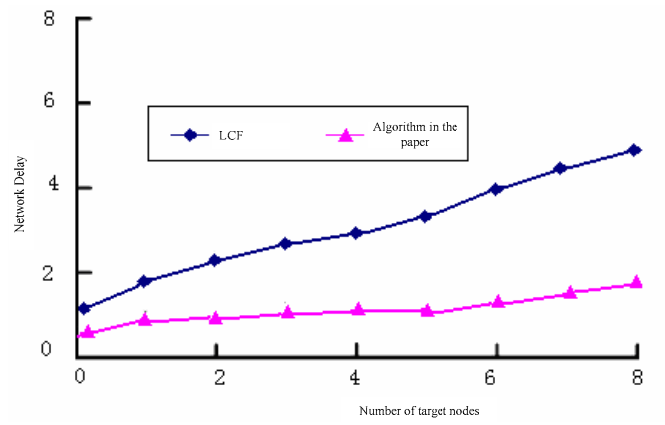

Figure 3. The comparison of the network delay

In figure 6, the delay of the traditional algorithm is larger than the proposed particles group algorithm and it is restricted by the target scale. It is not affected by the increase of nodes' scale and has good scalability.

\section{CONCLUSIONS}

The paper proposes an effective massive information communication method. The paths are sequenced according to the nodes capacity calculated by the effective weigh of the path in the network. The effective correcting algorithm is used to deal with the errors in the communication to tackle the problem of low efficiency. The experiment proves the algorithm proposed in the paper can greatly improve the efficiency of massive data communication in equivalent network which has high practical value.

\section{REFERENCES}

[1] Richard Bellman.A Markovian Decision Process[J].Indiana Univ. Math. J.6 No.4(1957), 679-684.

[2] Watkins, C.J.C.H..Learning from Delayed Rewards[].Ph.D. thesis, Cambridge University. 1989.

[3] Feilong Tang, Minyi Guo, Minglu Li,et all. Wireless Mesh Sensor Networks in Pervasive Environment: a Reliable Architecture and Routing Protocol[C], icppw, pp.72, 2007 International Conference on Parallel Processing Workshops (ICPPW 2007), 2007.

[4] Sanjay Shakkottai,Srikant R,Ness Shroff. Unreliable Sensor Grids: Coverage, Connectivity and Diameter[C].Proceedings of IEEE Infocom,2003:1073-1082.

[5] Vasilios Darlagiannis, Andreas Mauthe. Overlay Design Mechanisms for Heterogeneous,Large-Scale, Dynamic P2P Systems[J]. Journal of Network and Systems Management,Vo1.12, No.3, September2004. 